\title{
Parameter Calibration and Image Reconstruction of CT System
}

\author{
Mingxing Deng ${ }^{1}$, Xiaodong $\mathrm{Xie}^{1, *}$, Binke $\mathrm{Xia}^{2}$ and Tianhao $\mathrm{Li}^{2}$ \\ ${ }^{1}$ No. 4, Section 2, Jianshe North Road, Chenghua District, Chengdu, Sichuan, China \\ ${ }^{2}$ No. 2006, Xiyuan Avenue, Gaoxin West District, Chengdu, Sichuan, China \\ ${ }^{*}$ Corresponding author
}

\begin{abstract}
In this paper, a single target fitting optimization model for parameter calibration of CT system was built based on the least square principle in combination with the attenuation rule of $\mathrm{X}$-ray in media and the related theory of Radon transform. Applying this model to problem A of CUMCM in 2017, based on the method of grouping optimization, we applied genetic algorithm to solve the rotation center point and the spacing of detection units. The results obtained almost converge to the same value. Then under the calibrated CT system parameters, combined with the relevant theory of CT image reconstruction, we applied Fourier transform and Fourier Slice theorem to build a image reconstruction model of filtered back-projection. Based on the information received by the detector unit, the absorption rate information on every location was obtained, and the location as well as the shape of the medium on the tray were constructed. Finally, through the image reconstruction and comparison of the original data, the feasibility of the model is verified.
\end{abstract}

Keywords-Radon transform; group optimization; genetic algorithm; CT imaging; filtered back- projection

\section{INTRODUCTION}

In the case of not destroying the sample, the CT system can take advantage of the absorption characteristic of radiation energy by samples to form images of the biological tissue and engineering materials, so as to obtain the structural information inside the sample. A typical 2d CT system is shown in figure 1, parallel incoming X-ray is perpendicular to the detector plane, and each detector unit is regarded as a receiving point and they are equidistant. The relative position of the X-ray emitter and detector remains constant, and the whole transmitting and receiving system rotates 180 times counterclockwise around a fixed rotating center. For each X-ray direction, the radiation energy that has been absorbed and attenuated by the twodimensional medium was measured on the 512 equidistant detectors, and 180 groups of receiving information were obtained after the gain processing.

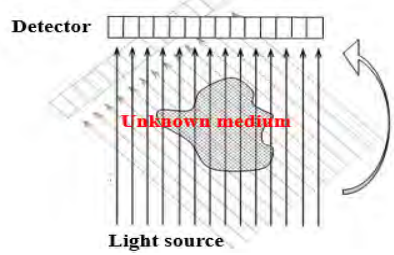

FIGURE I. SCHEMATIC DIAGRAM OF TWO-DIMENSIONAL PARALLEL BEAM CT SYSTEM
There are often errors in the installation of CT system, which affect the image quality. Therefore, it is necessary to calibrate the parameters of the installed CT system. That is, utilizing the samples with known structures to calibrate the parameters of the CT system.

The calibration of CT system parameters is the basis for the reconstruction of accurate images with the information measured by the detection unit. By using the main parameters of the calibrated CT system, the images of unknown media can be constructed accurately and relevant information can be obtained according to the information received by the detection unit. So it is necessary to build a general model to solve the relative parameters of CT system according to the absorption rate of calibration template and the receiving information of template. And then use the calibrated parameters for the image reconstruction of other materials and obtain the unknown information.

A specific example can be seen in problem A of the CUMCM in 2017[1]. In the first question, it gives 180 sets of receiving information obtained by an CT system whose parameters are unknown, requesting us to build a corresponding model to calibrate the relevant parameters of the CT system according to the information. In the second and third problems, In the second and third problems, the calibration parameters will be used to reconstruct the image of the medium according to the information of the unknown medium obtained by the detector.

In this paper, we propose a genera model for the parameter calibration and another model for the image reconstruction of the CT system, and then apply the models to a specific example in problem A of the CUMCM in 2017.

\section{PARAmetric Calibration Model of the CT System}

The structure of two-dimensional parallel beam CT system with rotation effect can be abstracted as shown below:

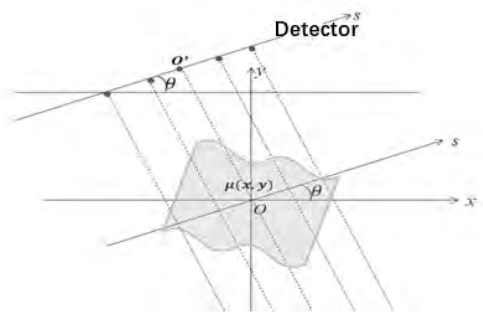

FIGURE II. SCHEMATIC DIAGRAM OF TWO-DIMENSIONAL PARALLEL BEAM CT SCANNING 
The s-axis is the one-dimensional coordinate axis of the detection unit, which can rotate around the rotating center. The origin is the intersection of the over-rotating center and the vertical line of the s-axis. It is easy to know that this point falls between the 256 and 257 detection units. The coordinate of the ith probe unit on s-axis from left to right is:

$$
s_{i}=\left(i-\frac{513}{2}\right) d
$$

Where $\mathrm{d}$ is the distance between the detection units.

When the rotation center of the CT system coincides with the coordinate origin of the coordinate system of the medium object coordinate system, if the current rotation Angle of the CT system is $\theta$, the detection line equation of the point $\mathrm{s}$ on the $\mathrm{s}$-axis can be written as:

$$
s=x \cos \theta+y \sin \theta
$$

It can be seen from the X-ray absorption law that, when the rotation Angle of CT system is $\theta$, the X-ray attenuation information received by the first detector can be recorded as[2]:

$$
p\left(s_{i}, \theta\right)=\iint \mu(x, y) \delta\left(x \cos \theta+y \sin \theta-s_{i}\right) d x d y
$$

The $\mu(x, y)$ is the adsorption rate of the medium object at the point $(x, y)$. When the center of rotation of the CT system does not coincide with the origin of coordinate system, the coordinate of the center of rotation point in this coordinate system is $(\mathrm{x} 0, \mathrm{y} 0)$, which can be obtained by coordinate transformation:

$$
\begin{gathered}
p\left(s_{i}, \theta\right)=\iint \mu\left(x-x_{0}, y-y_{0}\right) \delta\left[\left(x-x_{0}\right) \cos \theta+(y\right. \\
\left.\left.-y_{0}\right) \sin \theta-s_{i}\right] d x d y
\end{gathered}
$$

\section{A. Least Square Fitting Optimization Model}

For the total absorption rate $p_{i j}$, which is the obtained $\mathrm{i}_{\mathrm{th}}$ ray passing through the medium object in the $\mathrm{j}_{\text {th }}$ rotation, $p_{i j}=$ $p\left(s_{i}, \theta_{j}\right)$. The gain factor $\lambda$ is introduced here, so that the results reflected by $\lambda p_{i j}$ are the receiving information of the final output of the system. On this basis, the loss function $\mathrm{L}$ is constructed based on the least square principle, that is:

$$
L=\sum_{i} \sum_{j}\left(\lambda p_{i j}-t_{i j}\right)^{2}
$$

$t_{i j}$ represents the information measured by the ith detection unit in the jth rotation after the gain processing, that is, the $i_{\text {th }}$ row and jth column data in the attachment to the question.

The optimization model is built with the objective of minimizing the loss function as follows:

$$
\min _{\left(x_{0}, y_{0}\right), d, \lambda, \boldsymbol{\theta}} L=\sum_{i=1}^{512} \sum_{j=1}^{180}\left(\lambda p_{i j}-t_{i j}\right)^{2}
$$

$$
\text { s.t. }\left\{\begin{array}{rr}
-5<x_{0}<5 & \\
-5<y_{0}<5 & \\
0 \leq \theta_{j} \leq 360^{\circ} & j=1,2 \ldots 180 \\
\theta_{j+1}-\theta_{j}>0 & j=1,2 \ldots 179 \\
\lambda>0 & \\
d>0 &
\end{array}\right.
$$

Where, $(\mathrm{x} 0, \mathrm{y} 0)$ is the coordinate of the center point of rotation of the CT system, $\lambda$ is the gain factor, $\mathrm{d}$ is the distance between detectors, $\theta_{j}$ is the jth rotation angle of the CT system. The decision variables of the optimization model are $\mathrm{x} 0, \mathrm{y} 0, \lambda, \mathrm{d}$, $\left.\theta_{j}(\mathrm{j}=1,2) .180\right)$.

\section{B. The Method of Solving the Calibration Model}

According to the actual situation of the problem, the following adaptive solution pretreatment is carried out for the above fitting optimization model:

1) For the original image given by the problem, the pixel was expanded from $256 * 256$ to $1024 * 1024$ by bilinear interpolation to increase the accuracy of the solution.

2) With decision variables ( $x 0, y 0)$, d convert to the amount of image pixels ( $\mathrm{x} 0$ ', $\mathrm{y0}$ '), $\mathrm{d}$ ', to establish a continuous model on the question of discrete adaptive processing.

3) The received information data are equally grouped. All data are divided into 30 groups with 30 rotation angles as the interval between each group, and each group contained six rotation directions. In this way, the number of decision variables for each optimization solution is reduced and the computational complexity is reduced.

4) By analyzing the received information data, the constraint range of the rotation Angle is reduced so that the result can converge to the optimal solution faster.

Finally, the fitting optimization model after the adaptive correction of first problem is:

$$
\begin{gathered}
\min _{\left(x_{0}^{\prime}, y_{0}^{\prime}\right), d^{\prime}, \lambda, \boldsymbol{\theta}} L_{k}=\sum_{i=1}^{512} \sum_{\frac{j-k}{30}=0}^{5}\left(\lambda p_{i j}-t_{i j}\right)^{2} \\
k=1,2, \ldots 30 \\
\text { s.t. }\left\{\begin{array}{l}
-512<x_{0}^{\prime}<512 \\
-512<y_{0}^{\prime}<512 \\
25^{\circ}+j \leq \theta_{j} \leq 35^{\circ}+j \\
\lambda>0 \\
d^{\prime}>0
\end{array}\right.
\end{gathered}
$$

Where, $\mathrm{x}^{\prime}$ ' and $\mathrm{y}^{\prime}$ ' are pixel coordinates of the center point of rotation of the CT system, d' is the number of pixels between the detectors, $\theta_{j}$ are the jth rotation Angle of the CT system. The decision variables of the optimization model are $\mathrm{x}^{\prime}$ and $\mathrm{y} 0$ ', $\lambda$, $d^{\prime}$ and $\theta_{j}$.

$$
p_{i j}=p\left(s_{i}^{\prime}, \theta_{j}\right)
$$




$$
\begin{aligned}
& p_{i j}=\iint \mu\left(x^{\prime}-x_{0}^{\prime}, y^{\prime}-y_{0}^{\prime}\right) \delta\left[\left(x^{\prime}-x_{0}^{\prime}\right) \cos \theta\right. \\
& s_{i}^{\prime}=\left(i-\frac{513}{2}\right) d
\end{aligned}
$$

Considering that there are still many variables when 10 variables of the model are optimized, genetic algorithm[3], which performs well in multi-variable optimization, is adopted to solve the problem. We took the first set of data as an example to explain the solving process of genetic algorithm.

The decision variables we need to solve are $\left(x_{0}^{\prime}, y_{0}^{\prime}\right), d^{\prime}, \lambda$, $\left(\theta_{1}, \theta_{31}, \theta_{61}, \theta_{91}, \theta_{121}, \theta_{151}\right)$. The individual of ga is denoted as $\mathrm{g}$, and $\mathrm{g}$ is a set of values of decision variables. In this paper, real number coding is adopted. The kth generation population is called $G_{k}$, The ith individual in the kth generation population is denoted as $g_{i}^{\langle k\rangle}$.

The individual fitness function selects the loss function L1 in the above modified fitting optimization model, then the fitness of the individual $g_{i}^{\langle k\rangle}$ is recorded as $f_{i}^{\langle k\rangle}=L_{1}\left(g_{i}^{\langle k\rangle}\right)$.

The number of the population is 200 , and the number of iterations is 500. Matlab genetic algorithm tool kit was applied to solve the problem

\section{The Solving Result of Problem 1}

By solving 30 groups of optimization models, we get the means and variance of $x_{0}^{\prime}, y_{0}^{\prime}, \lambda, d^{\prime}$ are as follows:

TABLE I. THE SOLVING RESULT

\begin{tabular}{c|cc}
\hline \hline Decision variables & mean & variance \\
\hline$x_{0}{ }^{\prime}$ & -94.7891 & 0.3157 \\
$y_{0}{ }^{\prime}$ & 64.2381 & 0.1024 \\
$\lambda$ & 0.1745 & $8.4488 \times 10^{-7}$ \\
$d^{\prime}$ & 2.8314 & $1.3932 \times 10^{-4}$ \\
\hline \hline
\end{tabular}

We can see that the 30 groups of solving results are very concentrated, thus the means can be considered as the actual values of the decision variables. that:

The actual solving results are obtained by data conversion

$$
\begin{gathered}
x_{0}=\Delta_{0} \times(-64)=-9.2773 \mathrm{~mm} \\
y_{0}=\Delta_{0} \times 95=6.2500 \mathrm{~mm} \\
d=\Delta_{0} \times 2.8314=0.2765 \mathrm{~mm}
\end{gathered}
$$

Therefore, the position coordinate of the CT rotation center in the square tray is $(-9.2773,6.2500) \mathrm{mm}$, that is, $9.277 \mathrm{~mm}$ to the left from the center of the tray, $6.25 \mathrm{~mm}$ to the top, and $0.2765 \mathrm{~mm}$ to the detector unit. The initial rotation Angle of the CT system is 29.7893 degrees, which is roughly 180 degrees counterclockwise with 1 degree interval.

\section{TWO-DIMENSIONAL CT IMAgE RECONSTRUCTION MODEL}

Based on the central slice theorem of Fourier, a twodimensional CT image reconstruction model is established by filtering back projection method. According to the X-ray attenuation information of the CT system in 180 directions, the absorbance and geometric shape information of the measured substances can be reconstructed.

\section{A. Filtered Back-Projection Reconstruction Method}

In CT tomography imaging technology, the most direct way is by scanning information in all directions, directly through the accumulative method the projection to calculate the original image density (attenuation coefficient) of each pixel value. But it is in the rotating space reconstruction, so the accumulation will introduce stellate artifact directly, and the original image density zero points, different density at zero after reconstruction, then image distortion occurs. In order to overcome this phenomenon, we first interpolate and filter the projected data before projection, and then reverse-project the corrected data to obtain the image without false traces.

When image reconstruction of CT scan projection data is carried out by filtering back projection, we need to first select the response function of the filter[4]. Through the analysis of the reconstruction principle, we should know theoretically filter frequency response function is $|\omega|$, but actual system exists bandwidth, so in the design of filters, maximum frequency usually exists. The traditional method here generally adopts the ram-lak filter, and directly designs a band limit for the previous response function, as shown in figure 3 . However, this filter will generate Jibbs' phenomenon at high frequencies, which is the vibration of reconstructed image edges. Therefore, a more smooth method is adopted here, the shepp-logan filter[6]. The image of the response function is shown in figure 3 , which can be seen as $|\omega|$ with a smooth constraint.

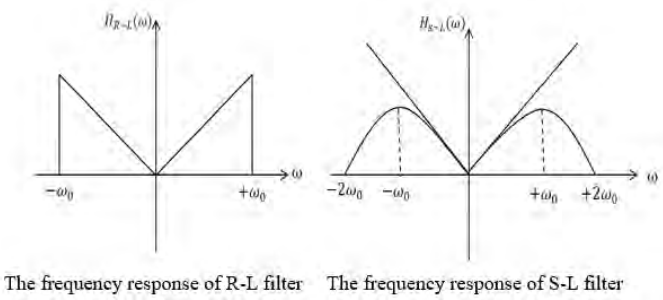

FIGURE III. THE FREQUENCY RESPONSE OF R-L AND S-L FILTER

The window function selected by shepp-logan filter is:

$$
W(\omega)=\operatorname{sinc}\left(\frac{\omega}{2 \omega_{0}}\right) \operatorname{rect}\left(\frac{\omega}{2 \omega_{0}}\right)
$$

Therefore, the frequency response function of s-l filter is:

$$
H_{S-L}(\omega)=|\omega| \operatorname{sinc}\left(\frac{\omega}{2 \omega_{0}}\right) \operatorname{rect}\left(\frac{\omega}{2 \omega_{0}}\right)
$$

The corresponding convolution function is: 


$$
h_{S-L}(s)=\frac{1}{2}\left(\frac{4 \omega_{0}}{\pi}\right)^{2} \frac{1-4 \omega_{0} s \cdot \sin \left(2 \pi \omega_{0} s\right)}{1-\left(4 \omega_{0} s\right)^{2}}
$$

The projection information in each direction is filtered through the filter, and the results are directly accumulated by the inverse projection method to obtain the original image's absorption distribution.

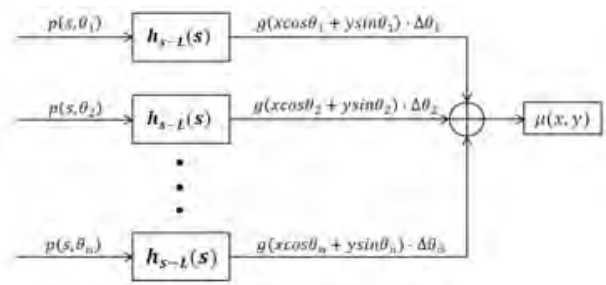

FIGURE IV. THE PROCESS OF FILTERING BACK-PROJECTION RECONSTRUCTION

\section{B. The Solution to the Model}

In order to obtain the actual attenuation information received by the detector, we need to restore the gain of the received information before the reconstruction of the filtering back projection. The data matrix of attenuation information is as follows:

$$
\begin{aligned}
P & =\left[\begin{array}{cccc}
p_{11} & p_{12} & \cdots & p_{1 p} \\
p_{21} & p_{22} & \cdots & p_{2 p} \\
\vdots & \vdots & \ddots & \vdots \\
p_{n 1} & p_{n 2} & \cdots & p_{n p}
\end{array}\right]=\frac{T}{\lambda} \\
& =\left[\begin{array}{cccc}
\frac{t_{11}}{\lambda} & \frac{t_{12}}{\lambda} & \cdots & \frac{t_{1 p}}{\lambda} \\
\frac{t_{21}}{\lambda} & \frac{t_{22}}{\lambda} & \cdots & \frac{t_{2 p}}{\lambda} \\
\vdots & \vdots & \ddots & \vdots \\
\frac{t_{n 1}}{\lambda} & \frac{t_{n 2}}{\lambda} & \cdots & \frac{t_{n p}}{\lambda}
\end{array}\right](n=512, p=180)
\end{aligned}
$$

The attenuation information is equivalent to the line integral of the absorbance on the detection line of the unit.

After the actual attenuation information received by the detector is obtained, the absorption distribution of the original image can be reconstructed when using the filtering back projection reconstruction method introduced above. Due to the center of rotation is not necessarily in the reconstruction of the geometric center of the object, so before the reconstruction, we needs to increase at the beginning and the end of the data matrix $\mathrm{P}$ each column on 100 units of zero filling it, to ensure that the image can be complete.

In the process of filtering back-projection reconstruction, we use MATLAB image processing tool kit[5] to implement the filtering back-projection method described above. Finally, we can reconstruct the matrix $\mathrm{M}(\mathrm{x}, \mathrm{y})$ based on the projection data $\mathrm{P}$, and this result is not the final distribution of absorptivity $\mu$ (x, y). Since we represent the absorptive distribution in a twodimensional image matrix, the absorptive value is actually the absorptive value of each pixel dot multiplied by the pixel width. The pixel width of the filtered back-projection reconstructed image is $\Delta_{0}^{\prime}=d$. So we have the following relationship:

$$
\begin{gathered}
\frac{M(x, y)}{\Delta_{0}^{\prime}}=\frac{\mu(x, y)}{\Delta_{0}} \\
\Delta_{0}=\frac{d}{d^{\prime}}
\end{gathered}
$$

Therefore

$$
\mu(x, y)=\frac{M(x, y)}{d^{\prime}} .
$$

At this point, the image pixel width is consistent with original image $\Delta_{0}$.

To ensure that the actual size of the image does not change, we use bilinear interpolation of MATLAB to expand the image $\mathrm{d}$ times. The image is then shifted $\frac{x_{0}}{d^{\prime}}$ horizontally and $\frac{y_{0}}{d^{\prime}}$ vertically. The final absorption distribution $\mu(x, y)$ can be obtained by cutting the image to $1024 \times 1024$. Because the problem requires a 256-by-256 distribution matrix, we used MATLAB to double linear interpolation to reduce the result by four times.

Finally, because the filter back projection reconstruction is a kind of numerical method, on the original image where the absorption rate should be 0 will be filled a small number, so in this paper, in dealing with a final result, the value less than 0.1 will be set to 0 to get the real situation of the absorption rate distribution.

\section{The Solving Result of Problem 2 and Problem 3}

At last, the media image of problem 2 and problem 3 were solved as follows:
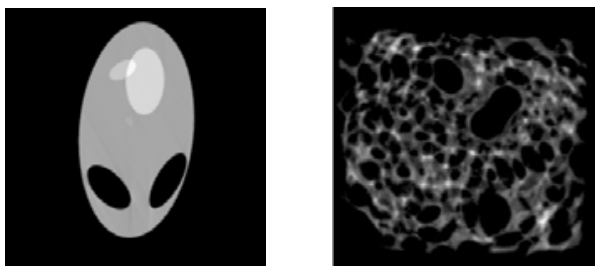

\section{Verification of Image Reconstruction Model}

Finally, we verify the feasibility of the model and algorithm of image reconstruction in this paper. We used the information of the known template that was detected by the CT system to reconstruct the image, and compared it with the actual template shape to show the feasibility of the reconstruction model and algorithm.

The following figure shows the comparison between the template image reconstructed by the CT image reconstruction algorithm and the original image :
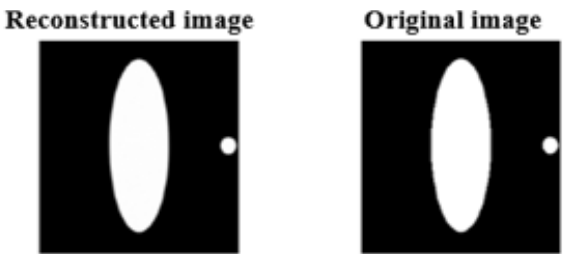
The location and geometric shape of the reconstruction results are consistent with the original image, and there are slight differences at the edge, but there is no impact on the overall problem. So the accuracy of the model is verified.

\section{PERORATION}

For the calibration of CT system parameters, based on the principle of X-ray attenuation, based on the principle of least squares, the loss function of the actual information received by the detection unit of the CT system and the theoretical calculation value is obtained, and the unknown parameter is used as the decision variable. Then the single objective optimization model is established.

For the above optimization model, it is very difficult to obtain a direct solution because of too many decision variables, or even to obtain the correct solution. Therefore, according to a certain rule, six groups of data are extracted from 180 sets of data at a time for grouping optimization. It is better to use the radon transform sine diagram to obtain the approximate initial value range of the rotation angle, which reduces the search range and increases the accuracy that makes it better to complete calibration of CT system parameters.

For the reconstruction of CT images, We adopt the filtering back-projection reconstruction method, which removes the starshaped artifacts that appear in the direct back-projection reconstruction algorithm, and obtains accurate reconstruction images and specific information of unknown media. Finally we use the known template in the problem to verify the feasibility of CT image reconstruction model.

\section{REFERENCES}

[1] http://www.mcm.edu.cn/html_cn/node/460baf68ab0ed0e1e557a0c79b1c 4648.html

[2] Xiao-Jian H U, Zhang C L. The application of Randon transformation in image segmentation and characteristic extraction[J]. Journal of Zhongkai University of Agriculture \& Technology, 2006.

[3] Khazaii J. Genetic Algorithm Optimization[M]// Advanced Decision Making for HVAC Engineers. Springer International Publishing, 2016.

[4] Qureshi S A, Mirza S M, Arif M. Inverse Radon Transform-Based Image Reconstruction using Various Frequency Domain Filters in Parallel Beam Transmission Tomography[C]// Engineering Sciences and Technology, 2005. SCONEST 2005. Student Conference on. IEEE, 2006:1-8.

[5] Gonzalez R C, Woods R E, Eddins S L. Digital Image Processing Using MATLAB[M]. Publishing House of Electronics Industry, 2009.

[6] Gach H M, Tanase C, Boada F. 2D \& 3D Shepp-Logan Phantom Standards for MRI[C]// International Conference on Systems Engineering. IEEE, 2008:521-526. 